WellBeing International

WBI Studies Repository

7-2002

\title{
Social Learning of a Novel Avoidance Task in the Guppy: Conformity and Social Release
}

Culum Brown

University of Cambridge

Kevin N. Laland

University of Cambridge

Follow this and additional works at: https://www.wellbeingintlstudiesrepository.org/acwp_asie

Part of the Animal Studies Commons, Comparative Psychology Commons, and the Other Animal

Sciences Commons

\section{Recommended Citation}

Brown, C., \& Laland, K. N. (2002). Social learning of a novel avoidance task in the guppy: conformity and social release. Animal Behaviour, 64(1), 41-47.

This material is brought to you for free and open access by WellBeing International. It has been accepted for inclusion by an authorized administrator of the WBI Studies Repository. For more information, please contact wbisr-info@wellbeingintl.org.

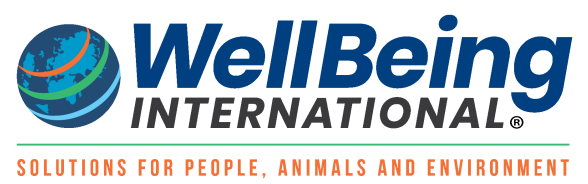




\title{
Social Learning of a Novel Avoidance Task in the Guppy: Conformity and Social Release
}

\author{
Culum Brown and Kevin N. Laland
}

University of Cambridge

\begin{abstract}
Studies of social learning suggest that many animals are disproportionately likely to adopt the behavior of the majority, and that this conformist transmission hinders the spread of novel behavioural variants. However, novel learned behaviour patterns regularly diffuse through animal populations. We propose a hypothesis, termed the 'social release hypothesis', that resolves these apparently conflicting findings by suggesting that animals are released from conforming to traditional behaviour in the absence of demonstrators. We investigated the role of pretrained, female demonstrator guppies, Poecilia reticulata, in influencing the escape response of untrained females to an artificial predator. Naïve 'observer' guppies were given the opportunity to follow trained demonstrators through an established escape route or escape independently via an alternative route. In the presence of demonstrators naïve fish overwhelmingly preferred to escape via the route taken by the demonstrators and escaped more quickly than fish in a control group with sham demonstrators. However, once the demonstrators were removed, the naïve fish were equally likely to use either escape route, despite once again escaping significantly faster than the controls. These findings are consistent with the social release hypothesis. Conformist social learning ensures that the behaviour of animals within a population is similar, and allows individuals rapidly to acquire locally adaptive information. However, innovations may spread as a consequence of social release.
\end{abstract}

Empirical and theoretical findings suggest that social learning allows animals to acquire locally adaptive information from conspecifics without having to pay some of the costs associated with asocial learning (Laland et al. 1993, 1996; Galef 1995; Giraldeau \& Caraco 2000). There is now considerable evidence that social learning enhances the foraging and antipredator behaviour of fish (for a review see Brown \& Laland 2001). Soical learning may result from the tendency of naïve individuals to approach conspecifics (Galef 1988), or via local enhancement, which is a common basis for shoal formation in fish. Laland \& Williams (1997) provided a clear demonstration of the social learning of foraging information in guppies, Poecilia reticulata. In addition, goldfish, Carasssius spp., minnows, Phoxinus phoxinus, and pollock, Pollachius spp., all forage more efficiently in social groups (Pitcher et al. 1982; Pitcher \& House 1987; Morgan 1988; Ryer \& Olla 1991). Furthermore, information about novel predators is learned and socially transmitted through shoals of minnows, sticklebacks, Gasterosteus aculeatus, zebra danios, Brachydanio rerio, and salmon, Salmo salar, (Magurran \& Higham 1988; Suboski et al. 1990; Ryer \& Olla 1991; Krause 1993; Pitcher \& Parrish 1993).

Fish within shoals are typically similar with respect to size, appearance and behaviour (Pitcher et al. 1985, 1986; Ranta et al. 1992; Pitcher \& Parrish 1993; McRobert \& Bradner 1998). It is widely believed that shoaling evolved largely in response to predation pressure, with individuals that look out of place or behaving unusually being preferentially targeted by predators (Parrish 1992). This 'oddity effect' 
generates selection on members of fish shoals to look and behave like others (Ohguchi 1981; Landeau \& Terborgh 1986; Pitcher \& Parrish 1993).

Social learning processes may further reinforce behavioural concordance within fish shoals. Theoretical analyses have found that in most circumstances where natural selection favours reliance on social learning, conformity is also favoured (Boyd \& Richerson 1985). These findings suggest that much animal social learning should involve individuals adopting the behaviour of the majority. Although few empirical studies have directly addressed these issues, evidence for conformist social learning has been observed in every species for which data exist (Day et al. 2001). Social learning in which the probability of adopting a pattern of behaviour increases with the proportion of demonstrators occurs in guppies (Sugita 1980; Laland \& Williams 1997; Lachlan et al. 1998), rats, Rattus norvegicus (Beck \& Galef 1989), and pigeons, Columba livia (Lefebvre \& Giraldeau 1994). Studies investigating social foraging in guppies suggest that conformity can hinder learning of a novel food-finding behaviour (Day et al. 2001). Both field and laboratory experiments report the maintenance of traditional behaviour of fish in foraging and mating site preferences, daytime schooling sites and migration routes (Helfman \& Schultz 1984; Warner 1988; Laland \& Williams 1997, 1998). However, evidence that fish have maintained socially acquired traditional behavior in the absence of demonstrators is comparatively scarce (Laland \& Williams 1997). This suggests that individual fish may relax their tendency to conform in the absence of clear demonstration.

One consequence of conformist social learning is that individuals should be less likely than chance to adopt novel behavioural variants. Empirical evidence from primates is consistent with this and suggests that most learned behavioural innovations fail to spread to other individuals, even if the behaviour appears to be of utility to the inventor, and the species concerned is known to be capable of social learning (Kummer \& Goodall 1985; Reader \& Laland 2001). None the less, there is also considerable evidence that novel behaviour patterns can spread through animal populations (Lefebvre \& Palameta 1988). This raises the question of how such diffusions are possible in animal populations that show conformity. This enigma would disappear if individual animals were to be released from conformity to social norms in the absence of clear demonstration, an explanation that we label the 'social release hypothesis'. Our aim in this study was to investigate this hypothesis by extending an earlier study by Brown \& Warburton (1999a).

Brown \& Warburton showed that social mechanisms enhanced the escape responses of rainbowfish, Melanotaenia duboulayi, to an approaching model trawl apparatus that contained a single escape route. Their observations suggested that fish that had successfully escaped provided others with a 'tip off' as to the location of the escape route. Similar conclusions were drawn by Laland \& Williams (1997) while investigating the social transmission of foraging route preferences in guppies.

We extended Brown \& Warburton's (1999a) study by investigating the social processes operating in shoals of guppies as they learn to avoid being trapped by a model trawl apparatus by swimming through one of two colour-marked holes in the trawl. Unlike Brown \& Warburton, we pretrained 'demonstrator' fish who provided demonstration to naïve observers as to which of two routes to use to escape from the trawl. We tested fish in two phases, first in the presence and then in the absence of conspecific demonstrators. If, as anticipated, conformity is instrumental in guiding the learning behaviour of guppies, once the demonstrators had been removed observers would be free of social constraints imposed upon them and would be equally likely to escape by either route. Provided that there was evidence that the observers had learned the escape behaviour, this would provide support for the social release hypothesis. Alternatively, observer fish may continue through habit to escape by using the route of their demonstrators even after the demonstrators' removal. This finding would be inconsistent with the social release hypothesis, and could lead to the establishment of stable traditions robust enough to be maintained in the absence of knowledgeable individuals. 


\section{METHODS}

We investigated the rate of learning of an avoidance task in 11 shoals of eight fish, each composed of four observer subjects and four pretrained demonstrators. The avoidance task required fish to swim through one of two differently coloured holes in a moving trawl to escape being trapped in a small gap between the side of the tank and the trawl net. The trawl procedure did not harm the subjects in any way; however, the fish were highly motivated to leave the small gap between the net and the wall of the aquarium for the open space on the other side of the net. Shoals of female guppies were tested first in the presence of demonstrator conspecifics, and subsequently in their absence. For each test phase we recorded the time taken for each fish to escape over 15 trials, the number of successful escapes and the escape route used. Demonstrators in the six 'experimental' groups were fish previously trained to escape, in three groups via a red-coloured hole and in three via a blue-coloured hole. In the remaining five 'control' groups there were untrained 'sham' demonstrators.

We used 100 female guppies: 44 as subjects and 56 as demonstrators. All fish were purchased from Neil Hardy Aquatica, London, U.K. We used a domestic strain of guppy, rather than the wild-type, as this allowed us to identify individual fish from natural colour markings, avoiding marking procedures. The guppies varied in size, and, as experiments have shown that fish size is a factor in shoaling preferences (Lachlan et al. 1998), we ensured each shoal consisted of a broad cross-section of different-sized guppies. We used female guppies because we did not wish sexual behaviour to interfere with the experimentation, and hence focused on single-sex populations. Female guppies tend to form the basis of shoals in the wild (Magurran \& Nowak 1991; Magurran \& Seghers 1994) and there is greater evidence for social learning in this sex (Dugatkin \& Godin 1992; Laland \& Williams 1997). However, social learning is by no means restricted to females (Sugita 1980; Reader \& Laland 2000).

All populations were housed in glass aquarium tanks $(61 \times 39 \mathrm{~cm}$ and $30 \mathrm{~cm}$ high), containing filtered and oxygenated water at $25^{\circ} \mathrm{C}$, to a depth of $33 \mathrm{~cm}$. Lighting was also controlled via timers, providing a 12:12 $\mathrm{h}$ dark: light ratio. The fish were fed once a day on commercial flake foods.

The experimental apparatus was contained in six glass aquarium tanks $(120 \times 39 \mathrm{~cm}$ and $30 \mathrm{~cm}$ high), containing filtered and oxygenated water at $25^{\circ} \mathrm{C}$, to a depth of $20 \mathrm{~cm}$. A model trawl apparatus similar to that used by Brown \& Warburton (1999a, b) was fitted to each tank. Rather than having a single centrally located escape hole in the trawl net, we cut two holes $(2 \times 2 \mathrm{~cm})$ in the top left and bottom right hand corners of the net $(2 \mathrm{~cm}$ in from each corner). Each hole was surrounded by a 1-cm rim of either red or blue crayon colouring (Fig. 1). During demonstrator training one of the holes was blocked by a piece of netting so that the demonstrators could be taught to take a particular escape route. The trawl net could be removed from the tank and set aside between trials.

\section{Procedure}

We randomly assigned five tanks of fish as control groups and divided the remaining six into three groups with demonstrators trained to escape via the red hole and three groups with demonstrators trained to escape via the blue hole. Six of the 10 fish within each group were randomly assigned to act as demonstrators and the remaining four became observers. We noted the pigmentation pattern of each fish to aid individual recognition. We did the experiment in two batches. In the first batch we tested two of each of the control, red-demonstration and blue-demonstration groups and in the second, which immediately followed the first, we tested three control groups and one each of the red and blue demonstration groups. In all cases the fish were transferred to the trawl tanks and allowed to settle for 3 days before the experiment. All test populations were housed in the test tanks throughout the experimental period partly to reduce handling stress but also because experiments have shown that the 
level of familiarity with the test environment may have significant impact on escape efficiency (Brown 2001).

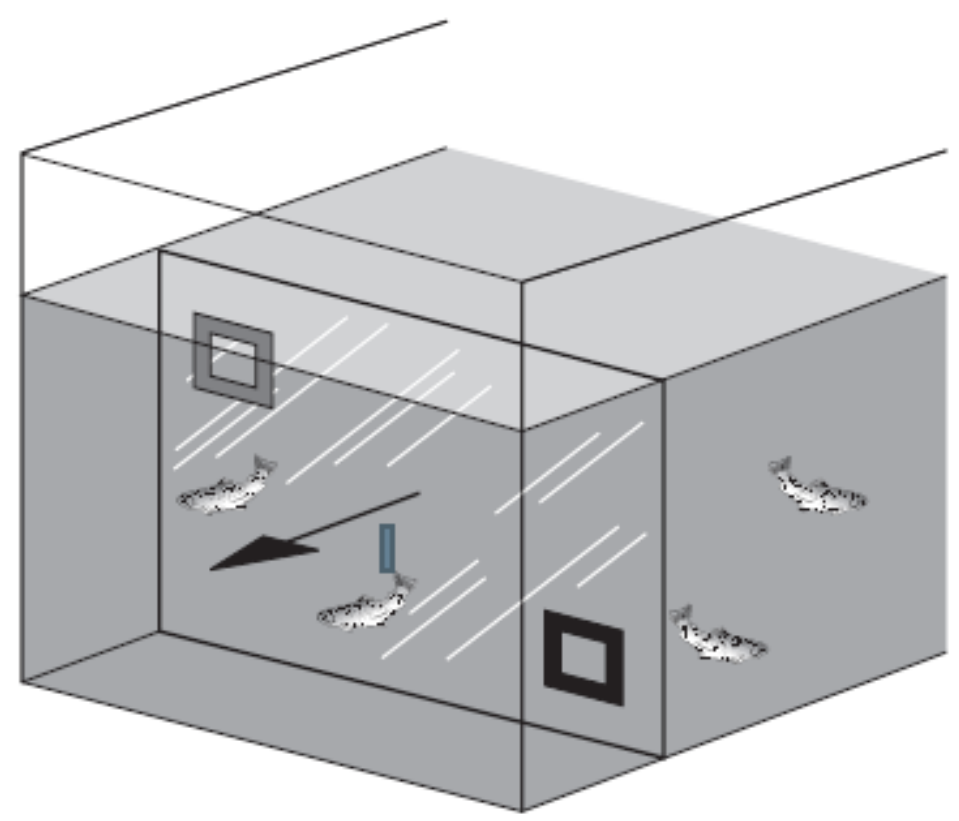

Figure 1. A diagram of the experimental apparatus showing the two alternative escape holes in the trawl net.

\section{Demonstrator training}

After the settling period, we removed the four observer fish from the test tank twice a day during morning and afternoon sessions while the demonstrators were exposed to five runs of the trawl. The observers were then reunited with their shoalmates. In the control groups we removed the observers for an equivalent length of time but the demonstrators were not exposed to the trawl. Each trawl run took $90 \mathrm{~s}$. The trawl net was set at the far end of the tank and over a 30-s period we slowly moved it down the length of the tank until it was $4 \mathrm{~cm}$ from the end, where we held it in position for a further $60 \mathrm{~s}$. To escape from the trawl, the fish had to locate and escape through one of the holes cut into the net. During this period one of the holes was blocked by a piece of netting. The red-trained demonstrators were forced to flee via the red hole and the blue-trained demonstrators via the blue hole. We recorded the time taken for each fish to escape through the target hole. After $90 \mathrm{~s}$ we lifted the net clear of the water and reset it at the far end of the tank. Between trials we had a 1-min interval. After 3 days of training, comprising 30 trials, the four demonstrators with the fastest escape times were selected and the remaining two were set aside. This ensured the demonstrators used later in the experiment were of high quality and clearly demonstrated the required task to the observer fish.

\section{Demonstrator-present phase}

Demonstrator training was followed by a demonstrator-present phase in which both the demonstrators and observers were exposed to the trawl while in the tank together. This occurred in three sets of five trials, with 1-min intertrial intervals, twice during the first day (one morning and one afternoon session) and once during the second day (morning only). During the demonstrator-present phase all of the fish 
were free to escape by either the red or blue hole. For each run we recorded both the escape latency of each fish and the colour of the hole through which it escaped.

\section{Demonstrator-absent phase}

During the afternoon on the second day we removed the demonstrators and tested the observers alone for five trials with 1-min intertrial intervals. The test period continued through to the third day where once again the observers were exposed to a morning and an afternoon set of five trials, giving 15 trials in total throughout the demonstrator-absent phase. Once again, we recorded the time taken for each fish to pass through the escape hole, the colour of the escape hole and the identity of the fish.

\section{Statistics}

Although guppies are loose shoalers and not schooling fish with coordinated movements, each fish cannot be treated as independent since fish within a shoal influence each other's movements to some degree and shoaling reduces the real number of degrees of freedom. Consequently, we adopted a hierarchically structured ANOVA design that included a 'tank' factor. This factor partialed out any between-tank variance. Where appropriate, data were log transformed to meet the required assumptions of homogeneity of variance and normality of residuals.
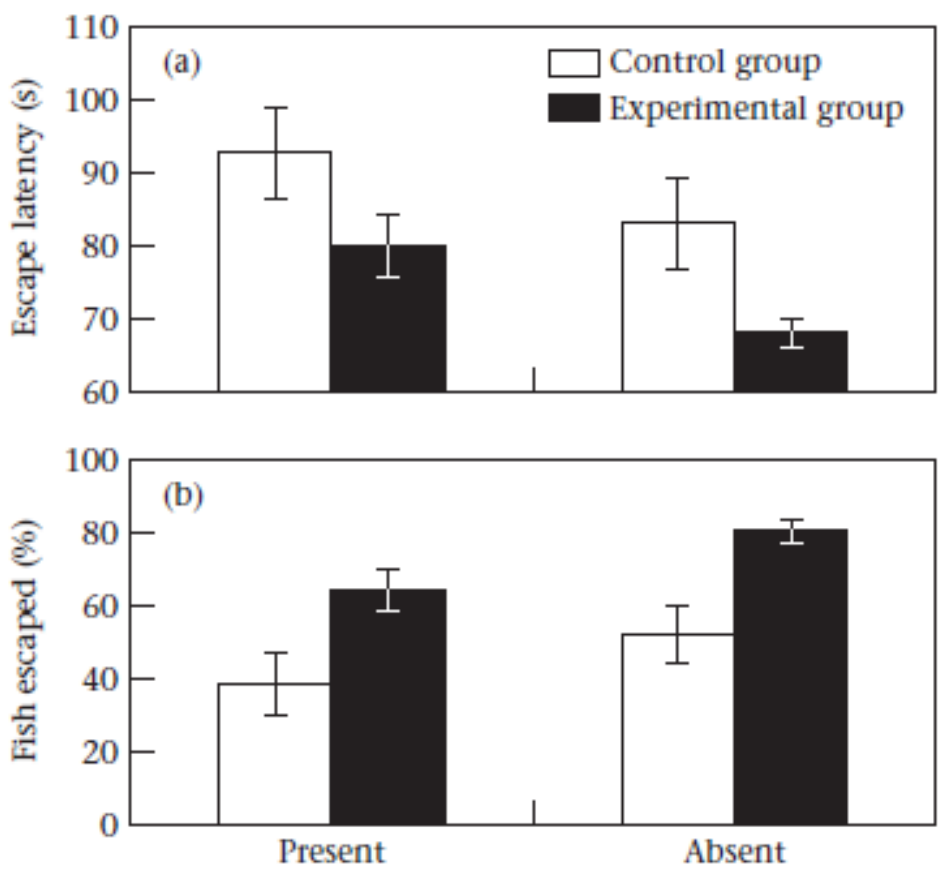

Figure 2. (a) The mean escape latency (s) for observer fish and (b) the percentage of observer fish that successfully escaped when demonstrators were present and absent. Means for experimental groups (fish with true demonstrators) represent the average for fish with both red- and blue-trained demonstrators. Control group refers to observers with sham demonstrators. Verical bars depict standard errors. $N=24$ experimental fish and 20 control fish. 


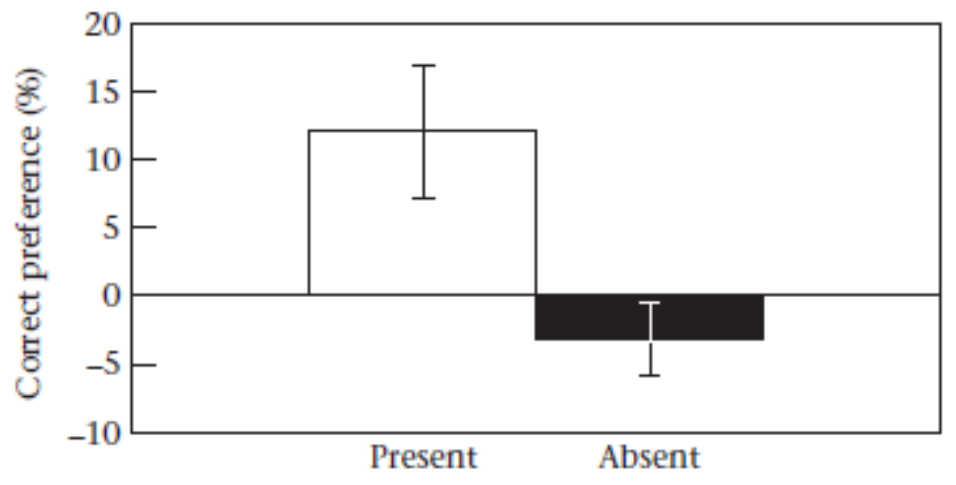

Figure 3. The preference for the correct escape route of observer fish that successfully escaped during the demonstrator-present and demonstrator-absent phase. $0 \%$ refers to $50: 50$ or no preference; $+5 \%$ refers to $55 \%$ use of correct hole or a $+5 \%$ preference for the correct escape route, etc. Means for experimental groups represent the average for fish with both red- and blue-trained demonstrators. Vertical bars depict standard errors. $N=24$ experimental fish.

\section{RESULTS}

\section{Demonstrator Performance}

Trained demonstrator fish were significantly faster at escaping the trawl than untrained demonstrators (ANOVA: $F_{1,33}=113.206, P<0.001$ ). Trained demonstrators had an average escape latency over the 15 trials of $62.4 \pm 3.7 \mathrm{~s}(\bar{X} \pm \mathrm{SE})$, whereas sham demonstrators had an average of $94.6 \pm 5.5 \mathrm{~s}$. Demonstrators trained to take the red route took this route significantly more frequently than those trained to take the blue route (ANOVA: $F_{1,18}=22.837, P<0.001$ ) and demonstrators trained to take the blue route took this route significantly more frequently than those trained to take the red route (ANOVA: $F_{1,18}=$ 122.474, $P<0.001$ ). Sham demonstrators had no escape route preference (ANOVA: $F_{1,15}=2.047$, NS). These findings confirm that the observers were exposed to appropriate demonstration.

\section{Observer Performance}

Observers exposed to real demonstrators escaped significantly faster $(\bar{X} \pm \mathrm{SE}=80 \pm 4.1 \mathrm{~s}$ with demonstrators and $69 \pm 2.9 \mathrm{~s}$ without demonstrators) than those exposed to sham demonstrators (93 \pm $6.1 \mathrm{~s}$ and $83 \pm 6.1 \mathrm{~s}$ ) during both the demonstrator-present and demonstrator-absent phases (repeated measures ANOVA: $F_{1,33}=18.958, P<0.001$; Fig. $2 a$ ). All observer fish improved their escape response significantly from the demonstrator-present to the demonstrator-absent phase (repeated measures ANOVA: $F_{1,33}=30.968, P<0.001$ ), but there was no group by test phase interaction (repeated measures ANOVA: $F_{1,33}=0.068, N S$ ). Performance for all observers improved across trials (repeated measures ANOVA: $\left.F_{14,462}=6.701, P<0.001\right)$, indicating that learning had taken place. There was a group by trial interaction (repeated measures ANOVA: $F_{14,462}=5.111, P<0.001$ ) reflecting the fact that the rate of learning differed between the control and experimental group observers. Observers with real demonstrators escaped faster from the first exposure so observers with sham demonstrators showed a greater improvement in escape latency over repeated trials. All trapped fish attempted to locate the escape holes in the trawl net. No fish chose to hide in the gap between the trawl net and the end of the aquarium. 
These findings were reinforced by data on the number of fish escaping over the 15 trials. Significantly fewer observers with sham demonstrators escaped from the net than observers exposed to trained demonstrators (repeated measures ANOVA: $F_{1,33}=62.486, P<0.001$; Fig. $2 \mathrm{~b}$ ). As expected from the escape latency data, the number of fish escaping in both groups improved from the first to the second phase (repeated measures ANOVA: $F_{1,33}=43.557, P<0.001$ ) but there was no group by test phase interaction (repeated measures ANOVA: $F_{1,33}=0.161, \mathrm{NS}$ ).

As predicted from the social release hypothesis, the observers trained with true demonstrators showed a preference for the trained colour during the demonstrator-present phase (ANOVA: $F_{1,18}=32.081, P<$ 0.001 ), but this preference was lost once the demonstrators were removed (ANOVA: $F_{1,18}=2.840$, NS; Fig. 3). During the demonstrator-present phase observers with demonstrators trained to take the red route took this route significantly more frequently than those with demonstrators trained to take the blue route (ANOVA: $F_{1,18}=112.108, P<0.001$ ). Observers with demonstrators trained to take the red route took the red route $63 \%$ of the time whereas observers with demonstrators trained to take the blue route took the red route on only $39 \%$ of occasions. However, during the demonstrator-absent phase observers with demonstrators trained to take the red route $(49 \%)$ tended to take this route slightly less frequently (although not significantly so) than those with demonstrators trained to take the blue route (56\%; ANOVA: $\left.F_{1,18}=1.323, \mathrm{NS}\right)$. Observers with demonstrators trained to take the blue route showed qualitatively similar behaviour. Observers in the control group had no escape route preference, during either the demonstrator-present phase (ANOVA: $F_{1,15}=0.151, \mathrm{NS}$ ) or during the demonstrator-absent phase (ANOVA: $F_{1,15}=0.654, \mathrm{NS}$ ).

\section{DISCUSSION}

Our results highlight the strong social influences that knowledgeable demonstrators can have on naïve observers. Observers exposed to trained demonstrators escaped more frequently and more quickly during both the demonstrator-present and demonstrator-absent phases than observers exposed to sham demonstrators. During the demonstrator-present phase observers in the experimental group followed their respective demonstrators through the hole that the demonstrators had been trained to use. In contrast, observers in the control group and their sham demonstrators had no escape route preference. Once the demonstrators were removed, however, any preference on the part of the observers for the escape route of their demonstrators was lost. The observers showed slightly more interest in the alternative escape route to that used by their demonstrators once the demonstrators were removed perhaps because of an increased tendency to explore the alternative escape route. Clearly the social influence was strong when the demonstrators were present, but once the demonstrators were suddenly removed, the social constraint was lost and the observers reverted to escaping by all available means.

One interpretation of the change in the observers' behaviour when the demonstrators were removed is that they had not learned to escape during the demonstrator-present phase, but merely shoaled with their escaping demonstrators. Two findings lead us to regard this as unlikely. First, despite the breakdown in the choice of escape route during the demonstrator-absent phase, observers in the experimental group still escaped more quickly and more frequently than control fish. Second, there was no deterioration in performance in the latency or frequency of escape among observers in the experimental group in the demonstrator-absent phase. Collectively, these findings allow us to dismiss the notion that no learning occurred during the demonstrator-present phase of the experiment. There is clear evidence that the observers in the experimental group improved their escape response by observing and interacting with their trained demonstrators during the first phase. When taken together these results are in accordance with the social release hypothesis described in the Introduction. It would seem that arbitrary aspects of the tradition of escape behaviour, such as the particular route chosen, were rapidly lost in the absence of the demonstrators, while functional aspects such as the enhanced latency to escape were preserved. 
Our casual impressions of the behaviour of the fish are in accord with this interpretation. During the intertrial interval of the demonstrator-present phase the fish were typically scattered throughout the tank. When the trawl began to move, trained demonstrators independently aligned themselves in front of their preferred escape hole, effectively forming a shoal, and calmly swam in front of the net. Once the net stopped, they simply turned and swam through the escape hole. The majority of the observers attempted to join the four demonstrators while the net was still in motion. By the time the net reached the far end, the observers also had to turn and escape through the closest available hole. In contrast, untrained demonstrators did not aggregate in front of the escape holes, showed little coordinated behaviour and provided no clear guidance to their observers. Fish trained with true demonstrators may have learned not only that there was an escape route but also that there was an alternative route, and proceeded in the demonstrator-absent phase to use both escape routes. Fish trained with sham demonstrators were still having difficulty finding any escape route during the latter phase.

Brown \& Warburton (1999a) attributed improved escape responses in larger shoals of fish to social facilitation; however, their experimental protocol did not allow them to specify the exact processes involved. Nevertheless, experiments examining the social transmission of foraging information in guppies have shown that the demonstrators are nearly always first to use a foraging route connecting a central compartment to a feeding chamber (Laland \& Williams 1997; Swaney et al. 2001). These and other experiments carried out in our laboratory have revealed that there are at least two ways in which trained demonstrator guppies can facilitate learning among observers (Swaney et al. 2001). Demonstrators may draw attention to the location of the hole and thus provide a 'tip off' to naïve observers, or observers may simply follow demonstrators directly along the route. During the demonstrator-present phase of the experiment, while the majority of observers either followed the demonstrators or a 'tip off' provided by them, some discovered the alternative route for themselves. All but one fish used both holes at some stage during the demonstrator-present phase. However, even after the alternative route had been discovered, the majority of the shoal continued to use the escape route favoured by the demonstrators. During the demonstrator-present phase, observers exposed to real demonstrators used the 'correct' escape route over $66 \%$ of the time. This is strong evidence for the social conformity effect whereby the behaviour of the majority compels the minority to conform (Warburton \& Lazarus 1991; Day et al. 2001). In this instance individual observers joined the shoal comprised of the four demonstrators. This conformity may be particularly strong in groups of animals that aggregate because of the threat of predation (Pitcher \& Parrish 1993).

In this instance the sudden loss of demonstrator influence caused a collapse in the maintenance of a social tradition (choosing the red or the blue escape route). If the demonstrators had been removed and replaced with naïve individuals one at a time then the route preference tradition might have been maintained. This has been found when transmission chain protocols were used (Curio et al. 1978; Laland \& Plotkin 1992; Galef \& Allen 1995; Laland \& Williams 1997, 1998). It appears that under these circumstances social traditions may be maintained in a population long after the founding individuals have been removed. Transmission chains probably more closely match what occurs in the wild where 'elders' or more knowledgeable individuals are gradually lost over time rather than all being lost in a single catastrophic event (e.g. Helfman \& Schultz 1984). However, where, because of migration or chance events, a clear pattern of majority behaviour is lost, there may be a release from this tendency to conform, aspects of the traditional behaviour may break down, and novel behavioural variants may spread (see Warner 1988). There are grounds to suspect that such events may be a general feature of animal traditions. In an analysis of primate behavioural innovation, Reader \& Laland (2001) reported that in approximately half of the cases where innovation occurred it was preceded by a major change in the environment, such as a period of food shortage, drought or habitat degradation. Such ecological 
challenges may leave established behaviour patterns ineffective or impossible, and thereby trigger social release.

Shoaling and other forms of aggregation by their very nature may encourage naïve members of a group rapidly to adopt locally adaptive and well-established behavior from more experienced individuals via social learning (Lachlan et al. 1998). Conformity and the oddity effect almost certainly inhibit the spread of novel behaviours through populations since individuals adopt the behavior of the majority rather than that of innovators. Among humans, compliance to social norms frequently continues in the absence of experienced or powerful group members, perhaps because processes present in humans but not other animals, such as enhanced episodic memory, communication and social cognition, may lead to rules and institutions that penalize nonconformists (Boyd \& Richerson 1985). In contrast, animal traditions may rarely reach the level of stability of human cultures, in part because of the frequent social release from conformity.

\section{Acknowledgments}

This research was supported by a Royal Society University Research Fellowship and Royal Society grant to K.N.L.

\section{References}

Beck, M. \& Galef, B. G. 1989. Social influences on the selection of a protein-sufficient diet by Norway rats Rattus norvegicus. Journal of Comparative Psychology, 103, 132-139.

Boyd, R. \& Richerson, P. J. 1985. Culture and Evolutionary Process. Chicago: University of Chicargo Press.

Brown, C. 2001. Familiarity with the test environment improves escape responses in the crimson spotted rainbowfish, Melanotaenia duboulayi. Animal Cognition, 4, 109-113.

Brown, C. \& Laland, K. N. 2001. Social learning and life skills training for hatchery reared fish. Journal of Fish Biology, 59, 471-493.

Brown, C. \& Warburton, K. 1999a. Social mechanisms enhance escape responses in the rainbowfish Melanotaenia duboulayi. Environmental Biology of Fishes, 56, 455-459.

Brown, C. \& Warburton, K. 1999b. Differences in timidity and escape responses between predator-naïve and predator-sympatric rainbowfish populations. Ethology, 105, 491-502.

Curio, E., Ernst, U. \& Vieth, W. 1978. The adaptive significance of avian mobbing II. Cultural transmission of enemy recognition in blackbirds: effectiveness and some constraints. Zeitschrift für Tierpsychologie, 48, 184-202.

Day, R., MacDonald, T., Brown, C., Laland, K. \& Reader, S. 2001. Interactions between shoal size and conformity in guppy social foraging. Animal Behaviour, 62, 917-925.

Dugatkin, L. A. \& Godin, J. G. J. 1992. Reversal of mate choice by copying in the guppy Poecilia reticulata. Proceedings of the Royal Society of London Series B, 249, 179-184.

Galef, B. G. Jr 1988. Imitation in animals: history, definition, and interpretation of data from the psychology laboratory. In: Social Learning: Psychological and Biological perspectives (Ed. by T. R. Zentall \& B. G. Galef Jr), pp. 3-28. Hillsdale, New Jersey: L. Erlbaum.

Galef, B. G. Jr 1995. Why behaviour patterns that animals learn socially are locally adaptive. Animal Behaviour, 49, 1325-1334.

Galef, B. G. Jr \& Allen, C. 1995. A new model system for studying behavioural traditions in animals. Animal Behaviour, 50, 705-717.

Giraldeau, L-A. \& Caraco, T. 2000. Social Foraging Theory. Princeton, New Jersey: Princeton University Press. 
Helfman, G. S. \& Schultz, E. T. 1984. Social tradition of behavioural traditions in a coral reef fish. Animal Behaviour, 32, 379-384.

Krause, J. 1993. Transmission of fright reaction between different species of fish. Behaviour, 127, 37-48.

Kummer, H. \& Goodall, J. 1985. Conditions of innovative behavior in primates. Philosophical Transactions of the Royal Society of London, Series B, 308, 203-214.

Lachlan, R. F., Crooks, L. \& Laland, K. N. 1998. Who follows whom? Shoaling preferences and social learning of foraging information in guppies. Animal Behaviour, 56, 181-190.

Laland, K. N. \& Plotkin, H. C. 1992. Further experimental analysis of the social learning and transmission of foraging information amongst Norway rats. Behavioural Processes, 27, 53-64.

Laland, K. N. \& Williams, K. 1997. Shoaling generates social learning of foraging information in guppies. Animal Behaviour, 53, 1161-1169.

Laland, K. N. \& Williams, K. 1998. Social transmission of maladaptive information in the guppy. Behavioral Ecology, 9, 493-499.

Laland, K. N., Richerson, P. J. \& Boyd, R. 1993. Animal social learning: towards a new theoretical approach. In: Perspectives in Ethiology 10. Behavior and Evolution (Ed. by P. P. G. Bateson, P. H. Klopfer \& N. S. Thompson), pp. 249-277. New York: Plenum.

Laland, K. N., Richerson, P. J. \& Boyd, R. 1996. Developing a theory of animal social learning. In: Social Learning in Animals: The Roots of Culture (Ed. by C. M. Heyes \& B. G. Galef Jr), pp. 129-154. London: Academic Press.

Landeau, L. \& Terborgh, J. 1986. Oddity and the 'confusion effect' in predation. Animal Behaviour, 345, 1372-1380.

Lefebvre, L. \& Giraldeau, L-A. 1994. Cultural transmission in pigeons is affected by the number of tutors and bystanders present. Animal Behaviour, 47, 331-337.

Lefebvre, L. \& Palameta, B. 1988. Mechanisms, ecology and population diffusion of socially learned food finding behavior in feral pigeons. In: Social Learning: Psychological and Biological Perspectives (Ed. by T. Zentall \& B. G. Galef), pp. 141-164. San Diego: Academic Press.

McRobert, S. P. \& Bradner, J. 1998. The influence of body coloration on shoaling preferences in fish. Animal Behaviour, 56, 611-615.

Magurran, A. E. \& Higham, A. 1988. Information transfer across fish shoals under predator threat. Ethology, 78, 153-158.

Magurran, A. E. \& Nowak, M. A. 1991. Another battle of the sexes: the consequences of sexual asymmetry in mating costs and predation risk in the guppy, Poecilia reticulata. Proceedings of the Royal Society of London, Series B, 246, 31-38.

Magurran, A. E. \& Seghers, B. H. 1994. Sexual conflict as a consequence of ecology: evidence from the guppy, Poecilia reticulata, populations in Trinidad. Proceedings of the Royal Society of London, Series $B, 255,31-36$.

Morgan, M. J. 1988. The influence of hunger, shoal size and predator presence on foraging in bluntnose minnows. Animal Behaviour, 36, 1317-1322.

Ohguchi, O. 1981. Prey density and selection against oddity by three-spined sticklebacks. Fortschritte der Verhaltensforschung, 23, 1-79.

Parrish, J. K. 1992. Do predators 'shape' fish schools: interaction between predators and their schooling prey. Netherlands Journal of Zoology, 42, 358-370.

Pitcher, T. J. \& House, A. C. 1987. Foraging rules for group feeders, area copying depends upon food density in shoaling goldfish. Ethology, 76, 161-167.

Pitcher, T. J. \& Parrish, J. 1993. The function of shoaling behaviour. In: The Behaviour of Teleost Fishes. 2nd edn (Ed. by T. J. Pitcher), pp. 363-439. London: Chapman \& Hall.

Pitcher, T. J., Magurran, A. E. \& Winfield, I. J. 1982. Fish in larger shoals find food faster. Behavioral Ecology and Sociobiology, 10, 149-151. 
Pitcher, T. J., Magurran, A. E. \& Edwards, J. I. 1985. Schooling mackerel and herring choose individuals of similar size. Marine Biology, 86, 411-416.

Pitcher, T. J., Green, D. A. \& Magurran, A. E. 1986. Dicing with death: predator inspection behaviour in minnow shoals. Journal of Fish Biology, 28, 439-448.

Ranta, E., Lindstro"m, K. \& Peuhkuri, N. 1992. Size matters when three-spined sticklebacks go to school. Animal Behaviour, 43, 160-162.

Reader, S. \& Laland, K. N. 2000. Diffusion of foraging innovations in the guppy. Animal Behaviour, 60, 175-180.

Reader, S. \& Laland, K. N. 2001. Primate innovation: sex, age and social rank differences. International Journal of Primatology, 22, 787-805.

Ryer, C. H. \& Olla, B. L. 1991. Information transfer and the facilitation and inhibition of feeding in a schooling fish. Environmental Biology of Fishes, 30, 317-323.

Suboski, M. D., Bain, S., Carty, A. E., McQuoid, L. M., Seelen, M. I. \& Seifert, M. 1990. Alarm reaction in acquisition and social transmission of simulated predator recognition by zebra danio fish Brachydanio rerio. Journal of Comparative Psychology, 104, 101-112.

Sugita, Y. 1980. Innovative choice behaviour in guppies. Japanese Psychological Research, 22, 7-12.

Swaney, W., Kendall, J., Capon, H., Brown, C. \& Laland, K. N. 2001. Familiarity facilitates social learning of foraging behaviour in guppies. Animal Behaviour, 62, 591-598.

Warburton, K. \& Lazarus, J. 1991. Tendency-distance models of social cohesion in animal groups. Journal of Theoretical Biology, 150, 473-488.

Warner, R. R. 1988. Traditionality of mating-site preferences in a coral reef fish. Nature, 335, 719-721. 